\title{
Estimation of Rigid Motion Parameters Using Moment Covariance Matrix
}

\author{
Chi Zhang ${ }^{\mathrm{a}}$, Jiangsheng You ${ }^{\mathrm{b}}$, Ming Zhang ${ }^{\mathrm{b}}$ and Patrick Wang ${ }^{\mathrm{a}}$ \\ ${ }^{a}$ College of Computer Science, Northeastern Univ., Boston, MA 02115, USA \\ ${ }^{\mathrm{b}}$ Medical OnLine Inc., Lexington, MA 02421, USA
}

\begin{abstract}
The estimation of rigid motion parameters has been investigated using Fourier transform and iterative techniques for over two decades. But the numerical efficiency remains a challenge. Especially for 3D case, there is no any analytical algorithm. In this work, we propose to use the moment covariance matrix to estimate the orthogonal transform (i.e., rigid motion in real world). This algorithm does not depend on the dimension of problem and is an analytical method. The numerical implementation is easy and efficient. The test results show that the proposed algorithm is accurate and well tolerant to noise variation.
\end{abstract}

Keywords: moments, covariance matrix, eigenvector and orthogonal transform.

\section{INTRODUCTION}

Estimation of rigid motion parameters has a variety of applications such as computer vision, clinical diagnosis and treatment through fusion of different images. Basically, there are two classes of methods: Fourier transform-based analytical algorithms [1] and various iterative algorithms [2]. Usually, these algorithms are efficient and accurate for two-dimensional case. But most of them are not applicable to three-dimensional case. Previously, moment is only used for determining some invariant patterns of images or objects [3], and has not been applied to estimating the transformation parameters. In this work, we propose to use the moment covariance matrix to estimate the transformation parameters. The results show that the new algorithm is quite efficient and accurate compared to the Fourier and iterative methods.

\section{ALGORITHM}

Let $f(x, y, z)$ represent any object, we define the first moments as

$$
\bar{x}(f)=\frac{\int f(x, y, z) x d x d y d z}{\int f(x, y, z) d x d y d z} \quad \bar{y}(f)=\frac{\int f(x, y, z) d y x d y d z}{\int f(x, y, z) d x d y d z} \quad \bar{z}(f)=\frac{\int f(x, y, z) z d x d y d z}{\int f(x, y, z) d x d y d z}
$$

Further, we define the second moments

$$
\begin{gathered}
\sigma_{x x}(f)=\int f(x, y, z)(x-\bar{x})^{2} d x d y d z \\
\sigma_{x y}(f)=\int f(x, y, z)(x-\bar{x})(y-\bar{y}) d x d y d z \\
\sigma_{x z}(f)=\int f(x, y, z)(x-\bar{x})(z-\bar{z}) d x d y d z
\end{gathered}
$$

and similarly, we may define $\sigma_{y x(f)}, \sigma_{y y(f)}, \sigma_{y z(f)}, \sigma_{z x(f)}, \sigma_{z y(f)}$ and $\sigma_{z z(f)}$. Now we have the covariance matrix 


$$
\left[\begin{array}{lll}
\sigma_{x x} & \sigma_{x y} & \sigma_{x z} \\
\sigma_{y x} & \sigma_{y y} & \sigma_{y z} \\
\sigma_{z x} & \sigma_{z y} & \sigma_{z z}
\end{array}\right]
$$

Calculate the eigenvectors of above symmetric matrix, we have orthogonal matrix $\$ Q(f) \$$ in which each column is one eigen vector.

Assume that $g(x, y, z)$ is the object after rigidly moving $f(x, y, z)$. Using the same analysis above, we have the orthogonal matrix $Q(g)$. Then the composite transform $Q^{T}(g) Q(f)$ is the orthogonal t ransform between $f(x, y, z)$ and $g(x, y, z)$.

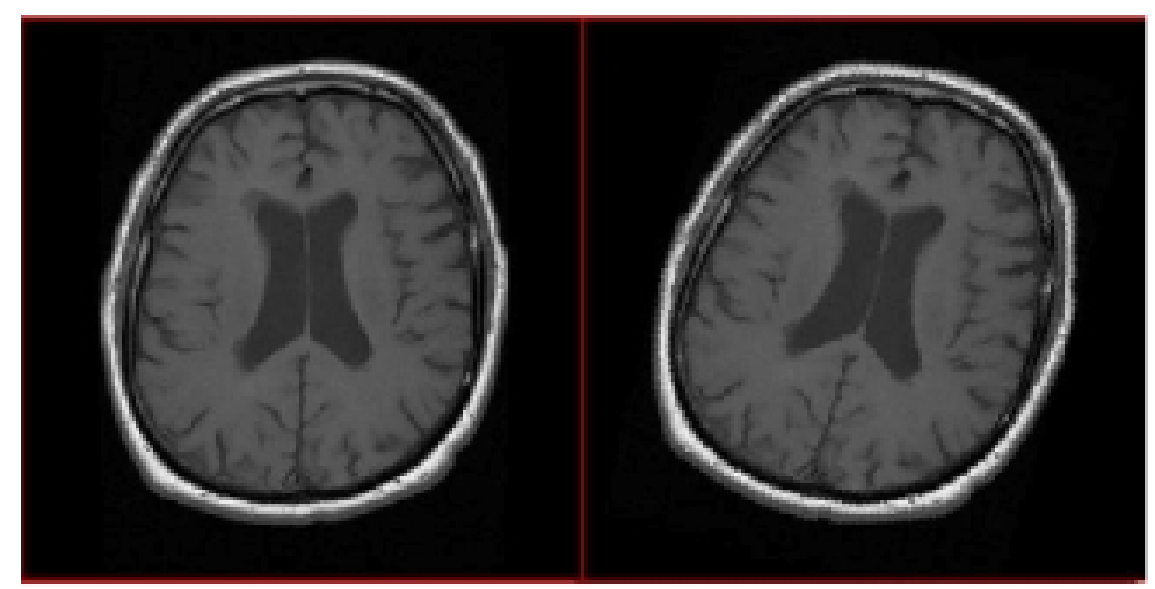

Figure 1

\section{NUMERICAL EVALUATION AND EXPERIMENTAL RESULTS}

The following are our experimental results. First, above algorithm is applied to two-dimensional (2D) medical images. Shown in figure 1, the left one is the original image, while the right one is the tilted image (rotated 15 degrees clockwise). Our algorithm gives the estimation value of 15.0659 degree. Next we apply our algorithm to threedimensional (3D) medical images. The matrices below show the actual transform orthogonal matrix and the estimated transform orthogonal matrix by our algorithm. The difference is less than $1 \%$.

2D:

$$
\left[\begin{array}{cc}
0.965926 & 0.258819 \\
-0.258819 & 0.965926
\end{array}\right] \quad\left[\begin{array}{ll}
0.965628 & 0.259929 \\
-0.259929 & 0.965628
\end{array}\right]
$$

3D:

$\left[\begin{array}{ccc}0.939693 & 0.330366 & 0.088521 \\ -0.342020 & 0.907673 & 0.243210 \\ 0.000000 & -0.258819 & 0.965926\end{array}\right] \quad\left[\begin{array}{lll}0.939700 & 0.330192 & 0.089088 \\ -0.341996 & 0.908377 & 0.240605 \\ -0.001479 & -0.256564 & 0.966526\end{array}\right]$




\section{CONCLUSIONS}

We have introduced a new methodology for estimating rigid motion parameters that a variety of applications including computer vision, medical image analysis, clinical diagnosis and treatment through fusion of different images. Basically, there are two classes of methods: Fourier transform-based analytical algorithms [1] and various iterative algorithms [2]. Usually, these algorithms are efficient and accurate for two-dimensional case. But most of them are not applicable to three-dimensional case. Previously, moment is only used for determining some invariant patterns of images or objects [3], and does not have applications to estimating the transformation parameters. In this research project, our proposed method uses the moment covariance matrix to estimate the transformation parameters. The results show that the new algorithm is quite efficient and accurate compared to the Fourier and iterative methods. In the future, we expect such method can also be widely applied to 3D medical imaging analysis, with robust 3D rotational display, similar to that of the 3D articulated object modeling, representation and visualization [4].

\section{REFERENCES}

1. E. D. Castro and C. Morandi, "Registration of translated and rotated images using finite Fourier transforms", IEEE Trans. Patt. Anal. Mach. Intel., vol. 9, pp. 700-703, 1987.

2. F. Maes, A. Collignon, D. Vandermeulen, G. Marchal and P. Suetens, "Multimodality image registration by maximization of mutual information", IEEE Trans. Medical Imaging, vol. 16, No. 2, pp.187-198, 1997.

3. M. K. Hu, "Visual pattern recognition by moment invariants", IRE Trans. Information Theory, vol. 8, pp. 179$187,1962$.

4. M. Yi and P. Wang, "3D Articulated Object Recognition in Virtual Environment”, ICPR'2000 (Int. Conf. on Pattern Recognition), September 2000, Barcelona, Spain. 